\title{
Scaffolding or Skeleton
}

Software Supports Student Multimedia Development Activities

\author{
Anne McDougall and Martin Boyle \\ Department of Science and Mathematics Education, University of Melbourne, Victoria, \\ Australia \\ a.mcdougall@edfac.unimelb.edu.au
}

Keywords: learner-centred learning, multimedia, research, teaching methods, case study

\begin{abstract}
A study investigated the use of software scaffolding for student design and construction of multimedia environments as a cross-curricular learning activity. The students (11 to 13 years) in a Melbourne, Australia, girls' school, worked in a technologically enriched learning environment and were experienced multimedia developers. The girls were provided with a preprepared scaffolding software package intended to challenge, stretch and support their learning. The scaffolding consisted of four aspects of Ancient Egyptian Mathematics: multiplication, The Horus-eye fractions, a hieroglyphic quiz and a potentially rich problem from the Rhind Papyrus. The girls were expected to take this scaffolding as a starting point and develop their own software package from it.
\end{abstract}

\section{INTRODUCTION AND DEFINITIONS}

This paper reports a study investigating the use of software scaffolding for student design and construction of multimedia environments as a crosscurricular learning activity. The subjects were students aged 11 to 13 years in a Melbourne, Australia, girls' school. The subjects developed multimedia software to teach students in the grade below them about aspects of Ancient Egyptian Mathematics. They were in a technologically enriched learning environment and were all experienced computer users. Each girl had her own notebook computer with the software necessary to produce multimedia packages. 
Scaffolding is "a means of providing a framework which challenges and stretches learners beyond their actual development" (Vygotsky 1978, p. 86) but which also "refers to the practice of providing just enough assistance (not too much, not too little) to help students succeed" (Meichenbaum 1998, p. 141). We consider the practice of scaffolding is especially useful in the context of computer use in learning environments. It must not be too clever, indeed it is often better for it to be unattractive for students, to build confidence and encourage the desire to improve, or even discard, the provided scaffolding.

The use of scaffolding for learning is not a new issue (Bruner 1982, Kaye 1982). Successful classroom teachers have used scaffolding techniques since there were classrooms. Scaffolding can be as simple and effective as encouraging words, through to the use of significant physical scaffolding described in the next section. Roehler and Cantlan (1997) emphasise the wide range of effective types and characteristics of scaffolding.

\section{SETTING THE SCENE FOR THE STUDY}

The work reported here forms part of a wider study of boys and girls in Melbourne schools as they develop multimedia software to illustrate various aspects of their curriculum. The software was developed in the Visual Basic programming environment. Other supporting software including graphics packages, image editors and linking support to the Internet, provided rich sources of visual and audio effects.

Working in such complex environments runs risks for the learner, and without support the inexperienced are soon lost. This is especially so for young learners in a professional programming area like Visual Basic, as opposed to more protected environments such as MicroWorlds where striking, if cognitively shallow, effects are easily achieved with the manipulative interface as opposed to developing projects under program code control.

\subsection{The scaffolding software described}

The project involved the students in the production of a multimedia software package, which would be used as a curriculum resource to introduce students in the grade below them to Ancient Egyptian mathematics. The subjects were provided with a pre-produced Visual Basic Project to scaffold their learning of both the production environment and the mathematical content, and a workbook with relevant information in which results could also be recorded. 
The scaffolding has been designed with an awareness that the level of support offered was crucial; it is vital that the complexity or cognitive demands of the task should not be reduced by the offered assistance. The scaffolding was menu driven and consisted of four main parts. Students were free to tackle the topics in any order they wished

\subsection{The nature of the topics}

The Ancient Egyptian multiplication algorithm. The multiplication algorithm (Figure 1) was included to provide a step-by-step mathematical process, offering the student the possibility of success merely by providing an explanation of the algorithm. It was of interest though to see whether the subjects would develop the Visual Basic form to incorporate their own presentational advances. It was not anticipated that the mathematical substance would be further developed, though the subjects were offered an avenue of expansion into binary number systems.

Horus-eye fractions. The Horus-eye fractions (Figure 2) were included to encourage the students to research the culture of Ancient Egypt beyond the mathematics. A mythical adventure underpins the fractions and the scaffolding was designed to encourage subjects to use various resources in exploring the implications of the myth for the ancient society and incorporate some of this information into their software.

A hieroglyph number quiz. The hieroglyph number quiz was designed to be the easiest of the activities to implement in an interest catching and dynamic environment. The students were encouraged to explore the number system of the Egyptians. Interest for the end users and the potential for interaction and dynamism in quizzing were also emphasised.

Powers of Seven. The most interesting of the mathematical content was to be found, well disguised, in the Powers of Seven section. Problem 79 of the British Museum's Rhind Mathematical Papyrus was introduced through the English 'Mother Goose' riddle 'On the way to St Ives'. This offered possibilities of expansion into international culture and also into a significant branch of modern mathematics. 


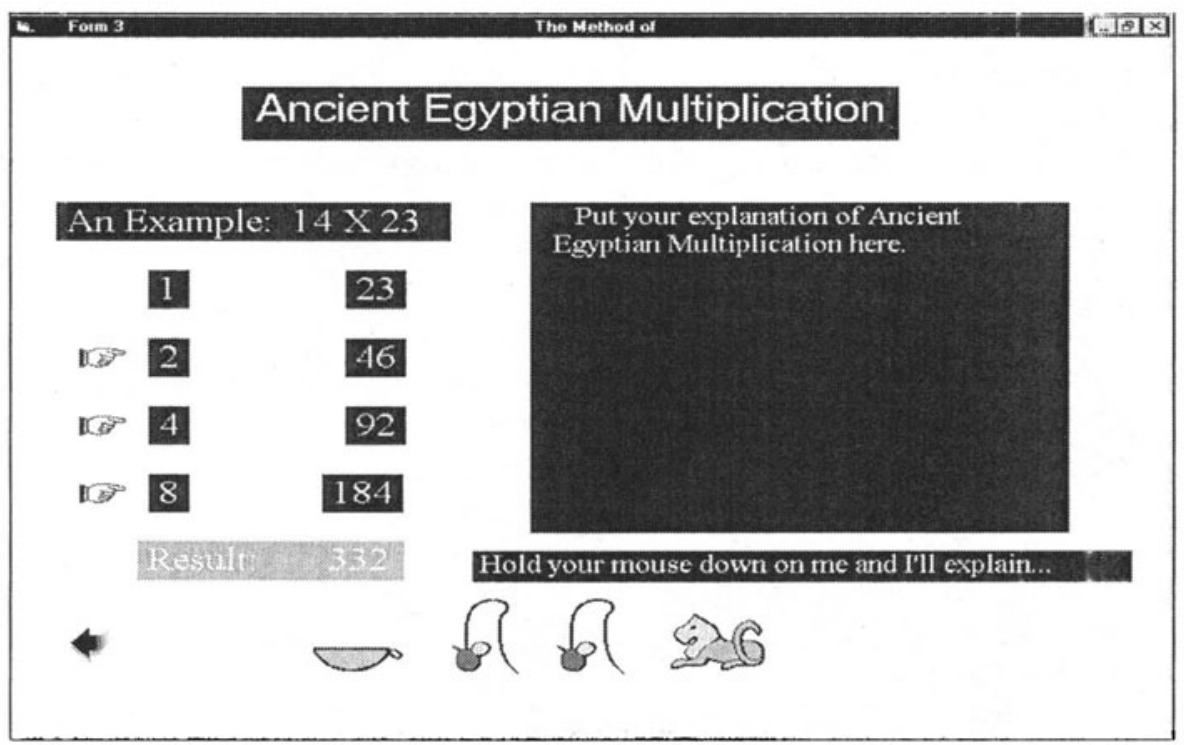

Figure 1. Ancient Egyptian Multiplication

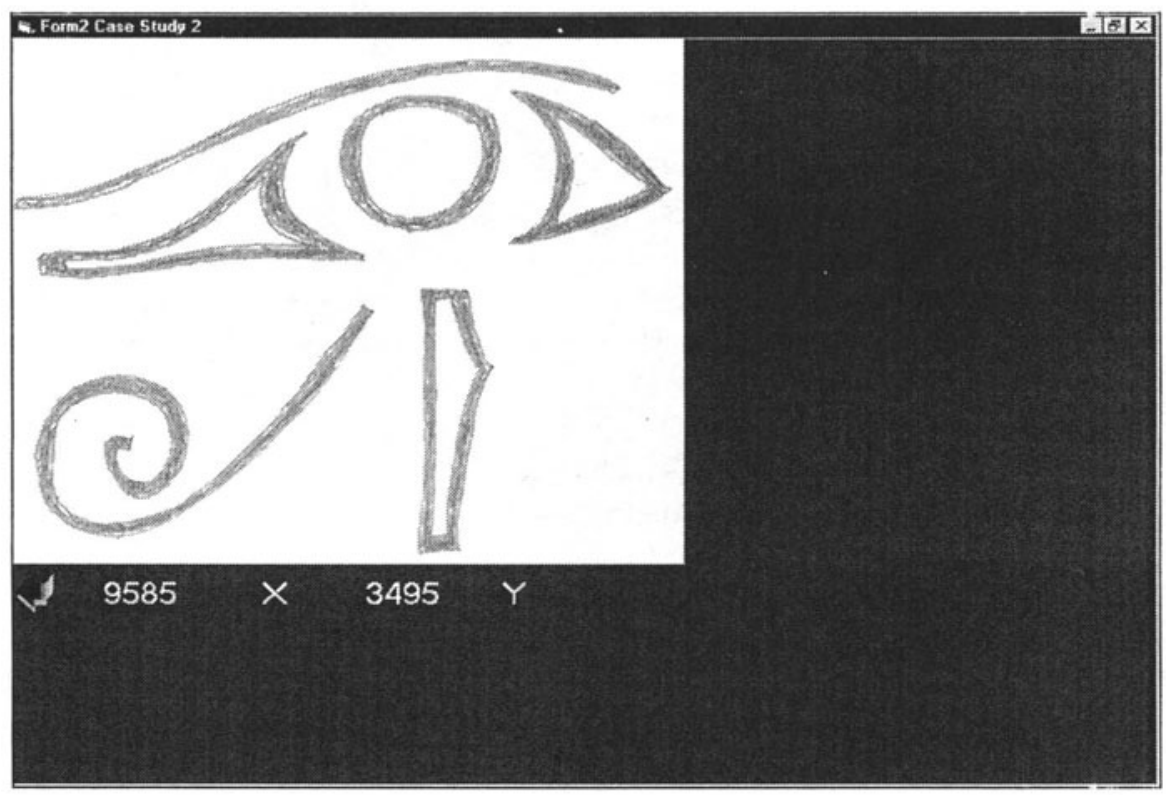

Figure 2. Horus-eye Fractions 


\section{METHODOLOGY}

The seven subjects were provided with copies of the scaffolding software and the workbook on Ancient Egyptian mathematics. They were to develop their own projects over six one-hour sessions. Their use of the scaffolding software was tracked for the first three sessions. The researcher maintained an observational $\log$, and interviewed each of the subjects about using the scaffolding software and the workbook. The combination of this data for four students is reported.

\section{RESULTS}

\subsection{Cherry's Story, Grade 5, 11 years old.}

\section{First session:}

$R$ : I see you're changing fonts.

Cherry: I don't want the same size! I want to do all my own thing!

$R$ : What do you think of the scaffolding software?

$C:$ I like it because it gives us an example and you can see what you want to do. You see it would take me ages to set this up. But maybe I don't like it because if we didn't have the software we wouldn't have this stuck in our minds forcing us really to do it this way.

Here we have a possible disadvantage of scaffolding. Cherry is reporting an over-influencing effect, feeling forced by the scaffolding to do things in a particular way.

\section{Second session:}

Whatever her concerns, Cherry was far from inhibited...

$R$ : You've made big changes.

$C$ : I'm doing the multiplication page first. And I want to get it completely finished. Look it's totally different to yours. The way you work the multiplication is the same of course, but as soon as I'd learnt how to do that from yours I changed it all so that it would be my own work.

\section{Third session:}

By this session Cherry was completely in charge of what she wanted from her software...

$R$ : You've changed the whole Horus-eye page?

$C$ : Yes, I wanted to set the graphics out in a completely different way.

$R$ : That didn't confuse you in working out the coordinates?

$C$ : No. It's all the same anyway, in the working out. 
From the outset Cherry was confident that she understood what was required and was happy to make substantial changes to establish her own interface.

\subsection{Eunice's Story, Grade 6, 12 years old}

\section{First session:}

$R$ : What do you think of the scaffolding software?

Eunice: It's OK...But maybe it's too much...it sort of says that I've got to do four things and maybe I'd do more or less. It will depend on what I want to do with the project. So this sort of makes me do what it wants. But it's OK. At least I know what to do, so I suppose I can do it in my own way later. Again the scaffolding is considered too constraining at the start.

\section{Second session:}

E: I've worked on the multiplication page. After I figured out how to do the multiplication from yours I changed it all. Now I think it looks real cool. See. Now Eunice is confident that she can cope with the content of the software and she is ready to break free from the scaffolding.

Now I'm working on the Horus-eye fractions. I've taken your graphic of the eye and divided it up into its six different parts and I've put them on different parts of the page. Now I've got to figure out what to do next to make it interesting.

Third session:

By the start of this session Eunice had worked through all four activities, making substantial changes to their appearance. There was also substantial change in the underlying code for all four even though the mathematics had not yet been done for two of them.

$R$ : You're happy doing all these changes even before you have explained all of the maths?

E: Yes. I know what I have to do so I'd rather just get on with it.

Eunice was very cautious at the start but when she found her feet she flew.

\subsection{Frances' Story, Grade 7, 13 years old}

\section{First session:}

$R$ : What do you think of the scaffolding software?

Francis: It's cool because I know what to do right from the start. I don't think I could have done this from the workbook because I wouldn't have known how to set up the pages to go from one to another. I'll learn, but it's good to get straight into the work without having to worry.

Frances immediately saw the complexity of this programming environment. She appreciated freedom to ignore the technical details, which would have taken the whole session to master unsupported. 


\section{Second session:}

F: I've worked on the multiplication. As soon as I understood what to do I made up my own example and put it in place of yours.

$R$ : I see you've left the screen more or less the same. It's your multiplication, but laid out much the same.

F: Yes. Your one's OK. I want to keep it so that I can concentrate on making sure that I explain the multiplication well. I'd rather put the time into making sure that people will understand.

An example of the developer putting the user first! Frances was determined to make sure the software worked well for her users and was happy to retain the example given in the scaffolding and devote her time to explanation.

\section{Third session:}

F: After what you said last time I've made so many changes to the Horus-eye page that I'm completely lost. See, I even change the picture so it's no longer a Horus-eye. I don't know what to do? Would it be possible to start again...but I've done my multiplication and I want to keep that.

$R$ : What we can do is delete just the Horus-eye page and then I can give you only that page from the scaffolding.

F: Yes please!

Frances started in her own cautious style but picked up erroneously from the researcher that she was expected to do more exciting things which she equated to making changes to the scaffolding. The subsequent departure from her normal work style could only be repaired to her satisfaction by starting again with the original software pages.

\subsection{Gale's Story, Grade 7, 13 Years old}

\section{First session:}

$R$ : What do you think of the scaffolding software?

Gale: I don't really know. Some things I like and some things I don't. It's not really our work. It's what you did. I would have to change all of it so that I can say that it's mine.

Gale is another student who need ownership of the software.

\section{Second session:}

G: I've done the multiplication. See, the screen is completely different to yours. I'm really happy to have my own version. Now I can tell people that it's my work. Of course I made sure I understood the multiplication you did before I changed it, but then I did my own one. Actually I did lots of examples to get a good one and I needed to get some extra graphics as mine is longer than yours.

Gale has not been able to wait to change the look of the software. She is much happier and more productive now that the software is hers.

$R$ : What's this you're doing now? 
G: I'm working on the Horus-eye fractions. I've taken the eye graphic and put it in its own image box. I think I want to move it around the screen, but I haven't done that yet.

Gale has decided on a complex area of development but she runs the risk of not being able to use the scaffolding code directly.

\section{Third session:}

Gale has replaced the Horus-eye graphic in its original position!

G: I see now why you gave us the scaffolding. I could never have worked out the coordinates for the Horus-eye fractions if I had to set up the whole thing myself. I've got it all working but now I want to animate the last part. Do you think that's a good idea?

Finally, Gale took a very 'common sense' approach of making sure she fully understood before making alterations or enhancements.

\section{CONCLUSIONS}

Though the scaffolding software was developed in the style of an individual it was quickly adapted by the subjects to use in their own learning style. The subjects soon realised that the process of personalising another developer's work was a learning experience in its own right.

It should be noted, though, that other than a page for recording coordinates, little or no use was made of the printed scaffolding workbook. The subjects became immediately engaged with the software.

The seven girls approached the scaffolding in quite different ways. For some it provided an initial framework which the girls extended, changed, and in some cases almost discarded as they developed their multimedia projects. For others it was more like a skeleton for their work, enabling various approaches to 'fleshing out' their projects, but providing a fundamental and lasting support structure around which they developed their own versions of the software.

The objections to the scaffolding were that the subjects did not feel initially that they were doing their own work and that the scaffolding in some way forced the subjects to work in a certain style.

The main advantages of the scaffolding were in providing inspiration for the subjects and, most importantly, providing time. The subjects had time to concentrate on the content of the project sure in the knowledge that the technical aspects of multi-media development were covered.

This small study supports the view that software scaffolding can be a valuable strategy for supporting learning in student multi-media development activates. The wider study, of which this work has been part, will investigate in more detail strategies for development of software 
scaffolding to cater for differences in student approaches to its use. Figures 3 and 4 below illustrate the girls' work. A typical re-working of the Horus-eye screen. The cursor is on the complex hieroglyph for $1 / 8$ at the top. Clickingon the black box leads to an informative Internet site

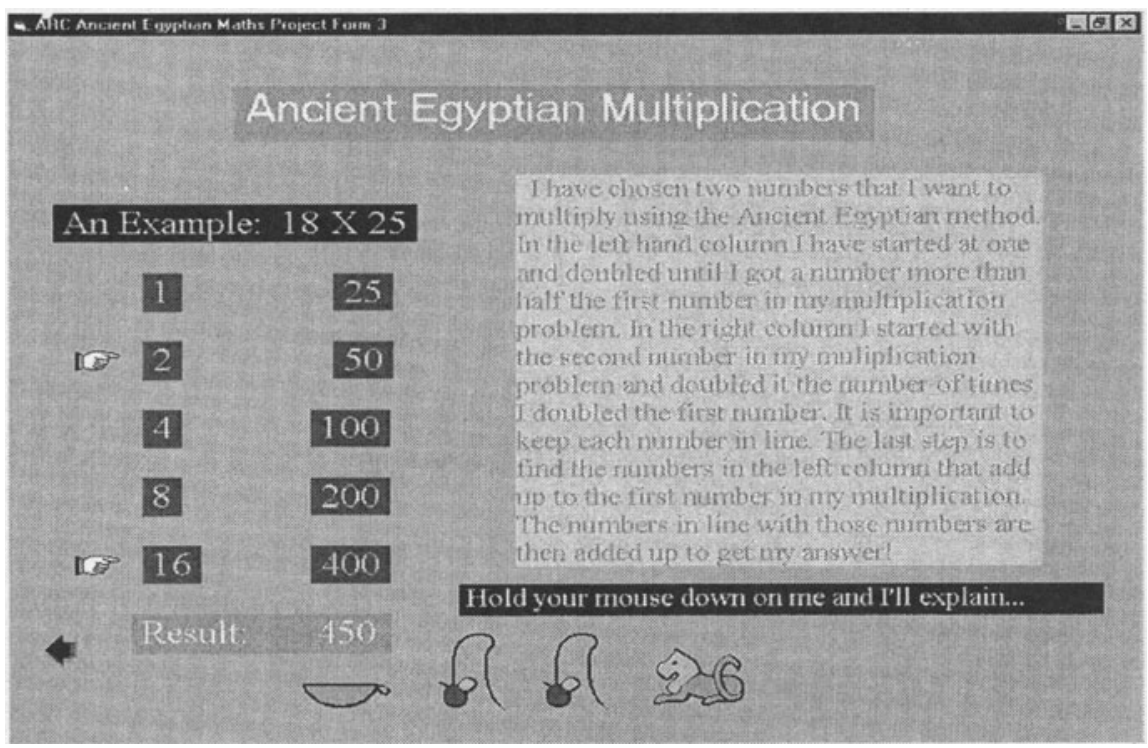

Figure 3. A Typical re-worked multiplication screen.

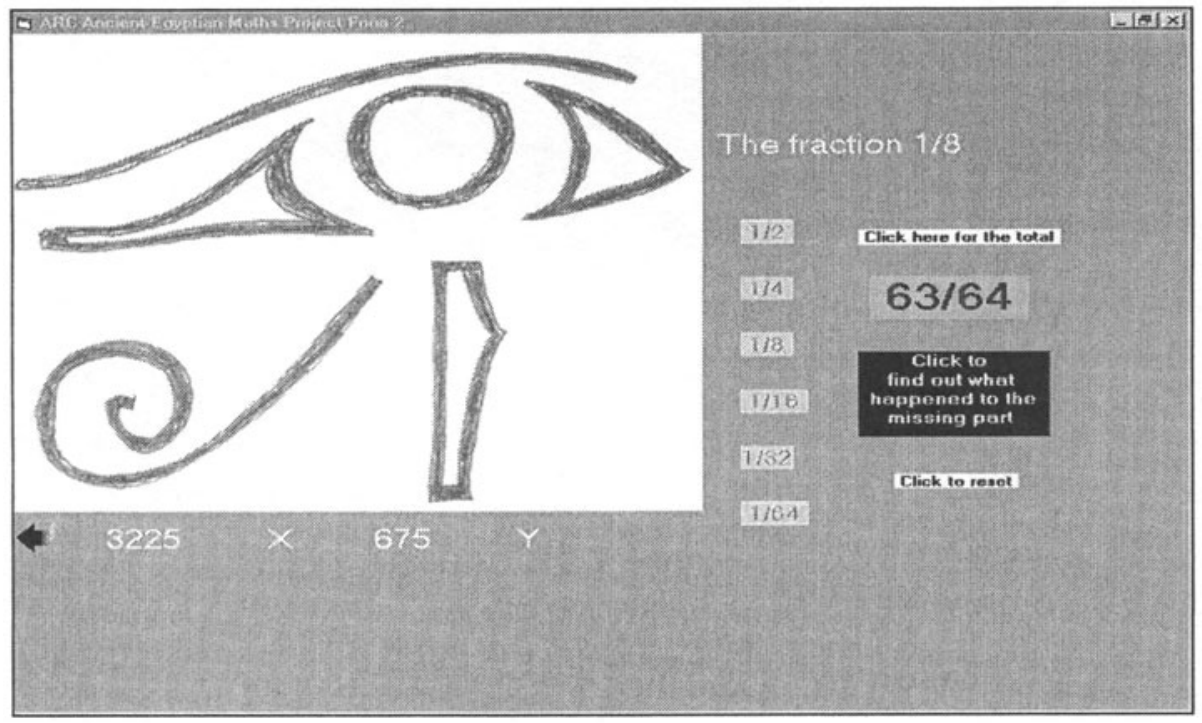

Figure4. A typical re-worked Horus-eye screen 


\section{REFERENCES}

Bruner, J. (1982) The Organisation of Action and the Nature of Adult-Infant Transaction. In The Analysis of Action, M. von Cranch and R. Harre (eds.), Cambridge University Press, Cambridge, UK, pp. 313-328.

Kaye, K. (1982) Organism, Apprentice and Person. In Social Interchange in Infancy, E. Tronick (ed.), University Park Press, Baltimore, MY, pp. 183-196.

Meichenbaum, D. and Biemiller, A. (1998) Nurturing Independent Learners. Brookline Books Cambridge, MA.

Roehler, L. and Cantlan, D. (1997) Scaffolding: A Powerful Tool in Social Constructivist Classrooms. In Scaffolding Student Learning: Instructional Approaches and Issues, $\mathrm{K}$. Hogan and M. Pressley (eds.), Brookline Books, Cambridge, MA.

Vygotsky, L. (1978) Mind in Society (Ed. M. Cole). Harvard University Press, Cambridge, MA.

\section{BIOGRAPHY}

Martin Boyle was educated in the UK and Australia and after extensive school teaching experience has spent the last five hears in teacher education. His research interests include developing learning environments to assist students who are failing in normal school mathematics. 